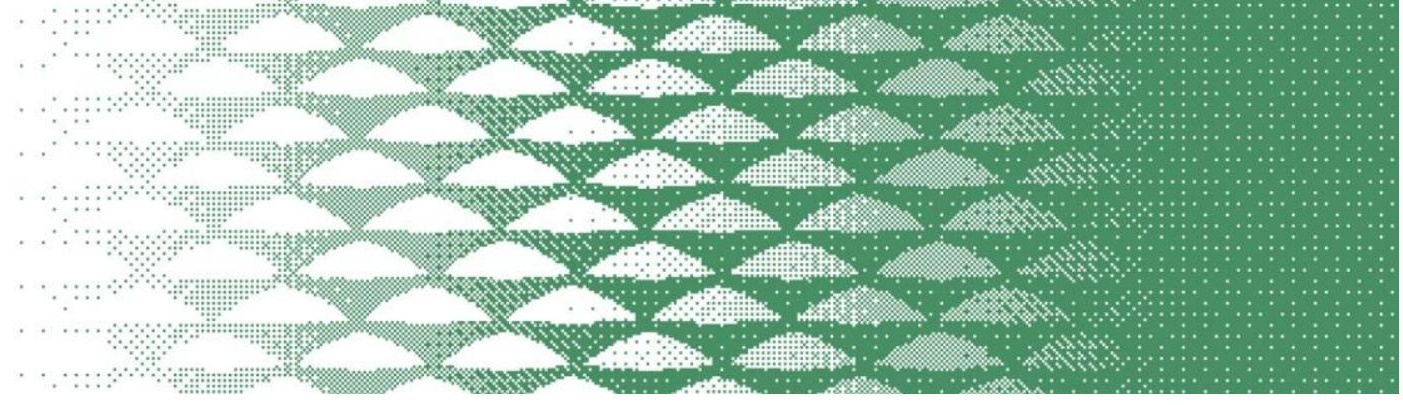

\title{
Têxteis Fabris: Identidade visual do acervo digital
}

\author{
Rodrigo dos Santos Souza'; \\ Sthephany de Oliveira Frazão; \\ Luciene Lehmkuhl ${ }^{3}$
}

\begin{abstract}
resumo:
O complexo fabril implementado na cidade de Rio Tinto pela Companhia de Tecidos Rio Tinto - CTRT teve papel significativo na cadeia produtiva têxtil nacional entre as décadas de 1920 e 1980, situando-se como um dos mais importantes do Brasil. Todos os processos vinculados ao desenvolvimento do produto têxtil ocorriam na própria cidade-fábrica que também absorveu influências europeias na arquitetura e na implementação inicial dos seus maquinários. Percebendo essas peculiaridades, o tema aqui destacado se tornou objeto de estudo para projetos de iniciação científica que nos últimos seis anos, têm registrado a memória do patrimônio material oriundo da cidade que vem passando por constantes processos de descaracterização. Com o intuito de disseminar a informação acerca do tema e propagar a história desse rico âmbito industrial brasileiro está sendo desenvolvido o acervo digital online "Têxteis Fabris" para abrigar os resultados das pesquisas realizadas. Por intermédio da plataforma Tainacan, o acervo disponibilizará, livremente para acesso e consulta, registros fotográficos dos principais elementos associados ao período de produção industrial da cidade. Dito isso, o texto aqui apresentado propõe abordar o processo de construção da identidade visual do acervo digital, elencando os elementos e etapas necessárias para a sua construção. Para isso, foi definida uma metodologia projetual que conseguisse gerir o desenvolvimento da identidade, bem como os testes necessários para sua validação. Como resultados são apresentados o logotipo que deverá estar associado ao repositório, ícones dos três principais temas que circundam o acervo, padrões que podem ser associados a imagens para divulgação, bem como a definição de um tema padrão a ser seguido dentro da plataforma Tainacan. Como conclusão se destaca a necessidade de considerar os testes para validar o trabalho até aqui executado.
\end{abstract}

palavras-chave: Rio Tinto; Têxteis Fabris; Identidade visual;

Espaço reservado para organização do congresso. 


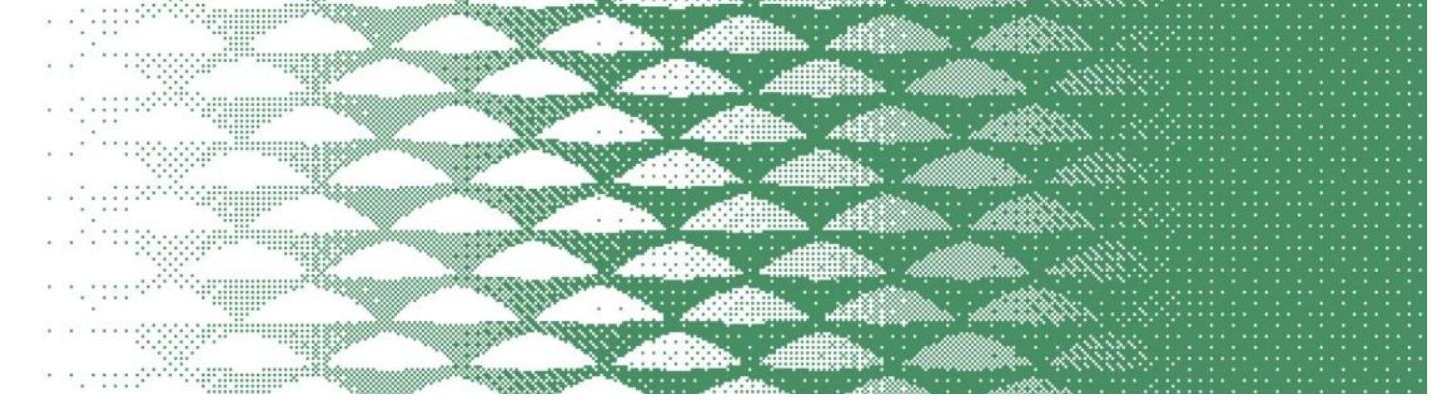

wordpress. Para isso, foi necessário o entendimento do próprio sistema e de como o acervo iria se comportar/comunicar no ambiente digital.

Por fim, o terceiro momento, ainda em construção, trata da finalização do acervo sob duas perspectivas: a primeira considerando a geração de uma identidade visual para o produto disponibilizado, com o objetivo de corporificar uma marca que apresente as características do projeto; e a segunda perspectiva consiste na validação do ambiente digital com a definição de protocolos e realização de testes com usuários. Dito isso, e entendendo a complexidade de cada perspectiva, o texto aqui apresentado propõe abordar somente o processo de elaboração da identidade visual elencando os elementos e etapas necessárias para a sua construção. A Figura 2 apresenta um esquema que sintetiza os três momentos descritos.

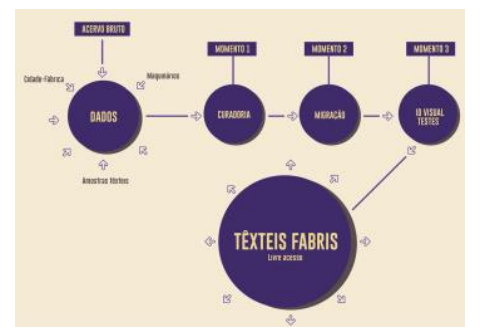

Figura 2 - Esquema síntese dos três momentos do projeto.

Fonte: Os autores (2021)

\section{Materiais e métodos}

Pensando no projeto como um todo e na fase em que se encontra para a finalização, a metodologia projetual utilizada foi escolhida por permitir tanto o desenvolvimento da identidade visual, quanto a execução dos testes para avaliação do acervo disponível na plataforma. As diretrizes elencadas dentro do método adotado sugerem a realização de reuniões em grupo; consultas com profissionais pesquisadores da área; pesquisas bibliográficas em bases de dados, bem como no conhecimento prévio associado ao tema em questão. Dessa perspectiva, é interessante destacar que podem ser coletadas informações no próprio acervo digital "Têxteis Fabris", assim como nos resultados de projetos anteriores que geraram produtos a partir dos elementos visuais que o compõem.

\subsection{O Tainacan como possibilidade para apresentação do acervo}

O Tainacan, software livre escolhido para abrigar o acervo, é um repositório para acervos digitais desenvolvido na Universidade Federal de Goiás, acompanhado do Ministério da Cultura e o Instituto Brasileiro de Museus (IBRAM) (MARTINS, 2017, p.6). Abrigam acervos dos mais distintos temas, enxergando a necessidade de conservar e preservar a memória usando como ferramenta a digitalização da informação (MARTINS, 2017). A proposta do Software Livre Tainacan surgiu da identificação da carência brasileira de políticas públicas de fomento à produção de repositórios digitais voltados à cultura. Viana (2005, p.3), assim como Lampert (2013, p.7), definem que os repositórios digitais cumprem a função de armazenar os objetos que apresentam formato digital, gerenciando e promovendo o acesso facilitado à informação. O repositório digital, como o acervo sobre a cidade e a fábrica de Rio Tinto, é proposto como uma fonte segura de informação sobre o tema e pretende servir às pesquisas e preservação dos elementos industriais associados à cultura material da cidade. A escolha do Tainacan para acomodação do projeto Têxteis Fabris deu-se pelo fato da plataforma caracterizar-se como um auxiliador na criação de repositórios digitais, possibilitando administrar e publicar as coleções de um acervo. 


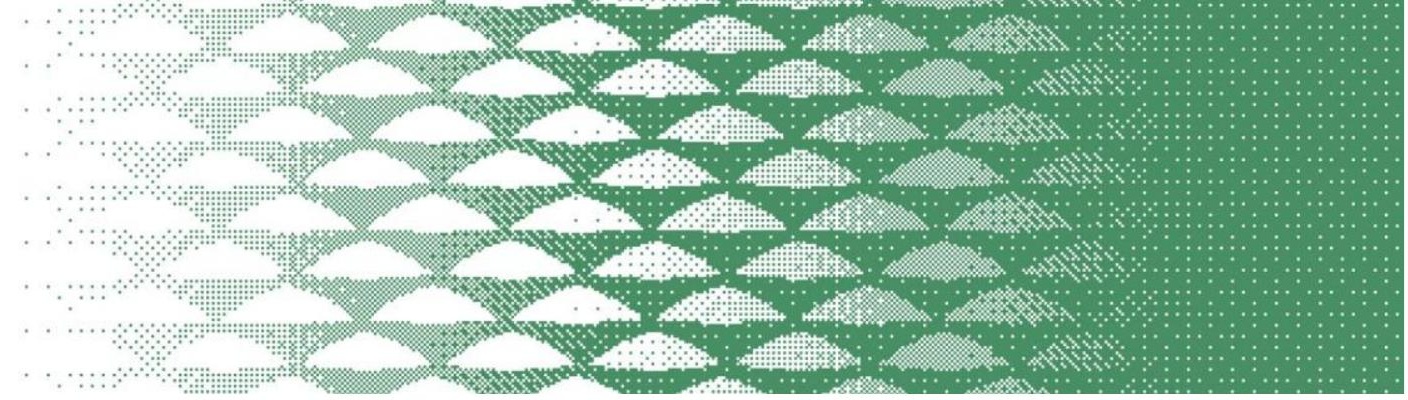

\subsection{Metodologia projetual}

O Guia de Orientação para Desenvolvimento de Projetos (GODP), se configura como uma metodologia para gestão projetual passível de associação ao fazer design, permitindo uma administração consciente dos processos e fases envolvidas. O GODP é dividido em oito etapas configuradas em estrutura cíclica (Figura 3) que se retroalimenta e permite a retomada de etapas, onde o fim de um projeto pode fomentar oportunidades para um outro trabalho (MERINO, 2014).

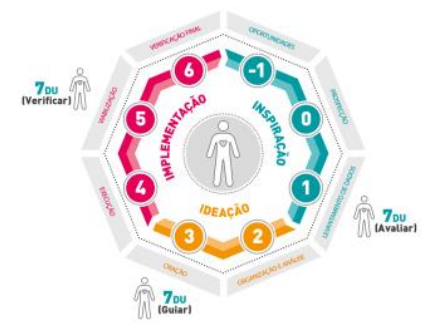

Figura 03 - Modelo de representação do GODP.

Fonte: E-book GODP. (http://ngd.ufsc.br/godp/) (2014)

A definição do GODP como metodologia para este projeto se dá pelo fato da identificação da oportunidade do trabalho aqui desenvolvido ter surgido mediante a conclusão de projetos de iniciação científica que também utilizaram o método para a identificação de suas oportunidades, possibilitando a continuação de estudos e interesses que já vinham sendo cultivados.

O método apresenta três grandes momentos, pensados de acordo com o Design Thinking (BROWN, 2009): inspiração, ideação e implementação. Dentro dos quais, encontram-se as oito etapas chave para orientar a progressão do trabalho proposto. Entretanto, a construção da identidade visual para o acervo foi associada aos dois primeiros momentos da metodologia, inspiração e ideação, os quais sedimentam e fornecem embasamento para a construção das ideias e proposição de alternativas.

Já o momento de implementação, que considera etapas como execução, viabilização e verificação final, pode ser associado a realização dos testes e validações do repositório digital como um todo, avaliando inclusive a identidade visual proposta. Portanto, o tema das últimas etapas da metodologia deverá ser abordado em um texto à parte, visando apresentar detalhadamente, os processos adotados e os resultados observados nas fases de teste e validação. A última etapa do GODP apresentada nesse artigo será a criação, associada ao momento de ideação, que consegue apresentar o processo de desenvolvimento da identidade visual.

Para além disso, o método destaca a necessidade de definir blocos de referência antes do início das etapas projetuais, tomados como aspectos norteadores capazes de contextualizar o público-alvo, o ambiente de utilização e o produto a ser desenvolvido. A partir dessa premissa, foram definidos como público-alvo pesquisadores, professores/alunos secundaristas, moradores da cidade de Rio Tinto e demais interessado no acervo; o produto em si é o acervo a ser disponibilizado livremente que deve contar com o desenvolvimento de uma identidade visual; como contexto definimos o ambiente virtual do Tainacan que receberá o acervo e permitirá que o usuário entre em contato com as informações disponibilizadas.

\section{Discussão e resultados}

Nesta seção será apresentado o desenvolvimento da metodologia projetual. 


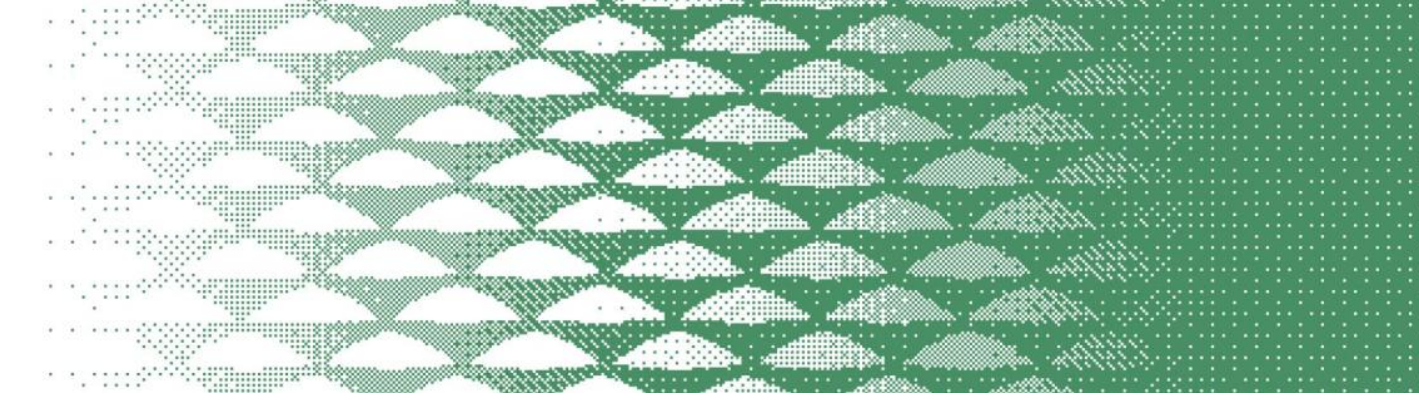

\subsection{Levantamento de dados}

A terceira etapa consiste no levantamento de dados levando em consideração a busca por informações pertinentes ao projeto, para isso, estudos bibliográficos se mostram fundamentais (MERINO, 2014, p.13). Desse modo, o entendimento sobre marca e identidade foram grandes norteadores para o desenvolvimento do projeto.

A marca, para Vásquez (2007, p.202), é um elemento visual que sinaliza e pode ir muito além dos signos visuais diretos, tendo características implícitas, podendo apresentar e representar uma série de associações emocionais e sociais. A marca define objetivos comunicacionais, deve significar e evidenciar, com o auxílio de elementos gráficos, uma identidade. Vasquez (2007) parte de uma perspectiva aplicada a empresas, que veem na construção da marca uma oportunidade de diferenciação dos concorrentes. A autora aponta que para se chegar a um resultado satisfatório devemos considerada a identidade conceitual da empresa, a qual fomenta a definição de uma missão (justifica a sua existência), visão (os objetivos que são almejados) e a cultura (símbolos, códigos e padrões) resultante das atividades realizadas pelo grupo que vão se aprimorando ao longo do tempo e que tem um fim comum (VÁSQUEZ, 2007, p.204). Logo, a identificação visual, para a autora, seria a concretização da identidade conceitual por meio de um sistema de signos/símbolos.

A marca, conforme apresenta Scherer e Scherer (2015, p.171), é um dos elementos mais importantes para a definição de uma identidade, e não se vincula necessariamente apenas a grupos que tenham fins lucrativos, tanto, que o projeto destes elementos vem sendo encarado com cuidado e rigor por cidades, entidades não governamentais, laboratórios de pesquisa acadêmicas ou, como no nosso caso, projetos vinculados a universidade. Para Raslan (2014, p.2) o consumidor/interlocutor deve conseguir identificar e relacionar os elementos contidos na marca com a empresa/grupo. Para isso, esses símbolos devem ser escolhidos com base no tipo de produto/serviço oferecido e na identidade do grupo, evitando assim confusões, dispersões e suscitando uma nitidez relacional entre a marca, identidade e visualidade. Desse modo, mesmo não sendo uma empresa, é importante para o desenvolvimento do projeto que haja clareza quanto aos seus objetivos e resultados esperados, então, considerar e apresentar uma missão, visão e cultura pode auxiliar nesse processo.

Como Missão propomos valorizar e preservar a cultura material e imaterial do território riotintense para que futuros pesquisadores, moradores da região e demais interessados tenham acesso a esse rico patrimônio de forma facilitada; a Visão almeja conseguir compartilhar, com o maior número de pessoas, os resultados encontrados durante o desenvolvimento dos projetos de iniciação científica; e a Cultura quer garantir o direito de futuras gerações conhecerem o patrimônio histórico-cultural, para isso fazendo uso e divulgando seus elementos simbólicos.

Um ponto interessante a se destacar é o ambiente de aplicação da marca. No ambiente virtual, além de conferir personalidade a um nome, o diferenciando dos semelhantes, um logotipo pode receber múltiplas funções que interferem no sistema computacional utilizado. É o que considera Passos (2014, p.12-13), segundo o autor há que se pensar cuidadosamente a relação dos elementos gráficos e a sua disposição dentro de um site, pois muitas vezes esses elementos podem ser interpretados como localizadores. Por exemplo, muitas vezes o logotipo serve como um botão para voltar ao início do site. Entendendo as relações diretas e explícitas que uma logo/marca pode exaltar, partirmos para a próxima fase do método.

\subsection{Organização e análise}

A etapa organização e análise propõem a compreensão das informações coletadas nas etapas anteriores e a definição de requisitos para o projeto. Com isso, podemos considerar o interesse na construção de um logotipo que identifique e incorpore características do projeto, utilizando os símbolos gráficos já destacados ao longo do estudo como ponto de partida; o desenvolvimento de ícones gráficos descritores dos três grupos de coleções, a partir do acervo, elencando um elemento síntese para cada grupo e, ainda, a definição de tipografias para textos e títulos. 


\title{
JOP DESIGN
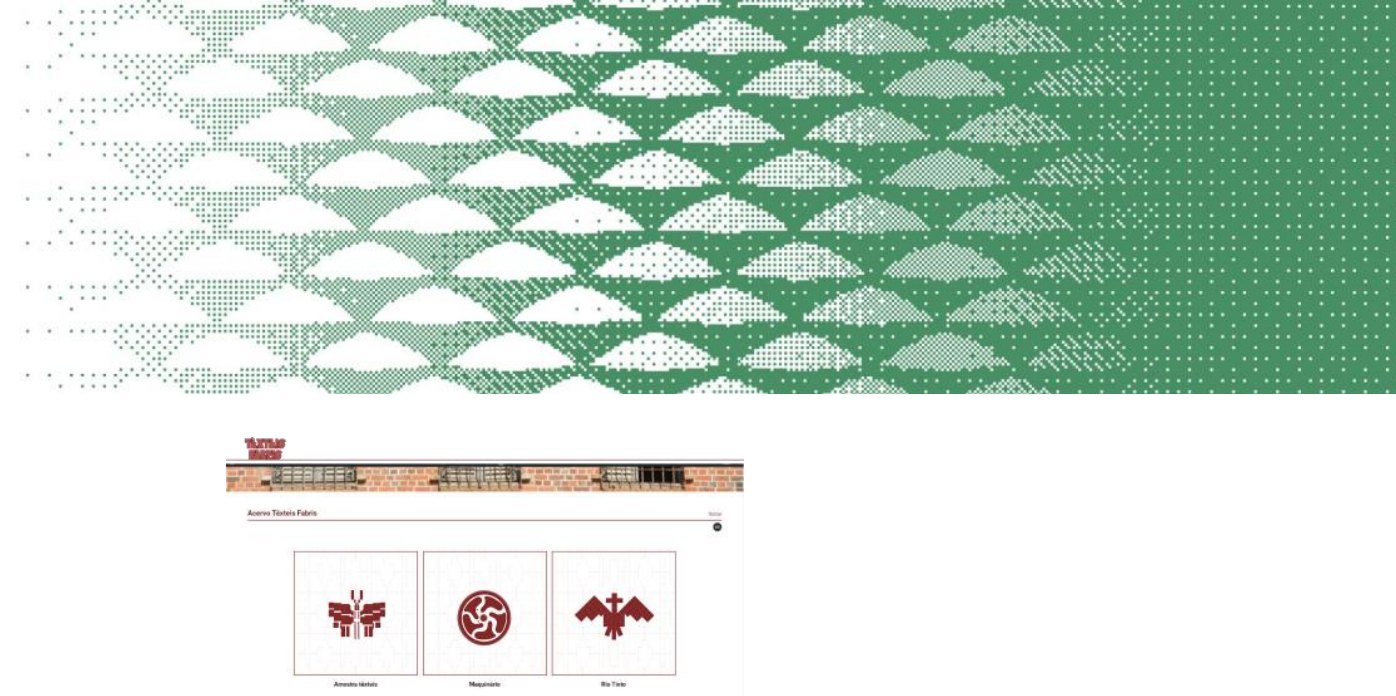

Figura 11 - Aplicação dos elementos desenvolvidos dentro do repositório.

Fonte: Os autores (2021)

\section{Conclusão}

O texto aqui proposto apresentou as primeiras etapas e momentos para a finalização do repositório digital Têxteis Fabris que consideraram o desenvolvimento de uma identidade visual para o acervo. Como resultados, chegamos a uma proposta de logotipo, ícones para serem associados aos principais temas do acervo, padrões passíveis de aplicação em banners e imagens para divulgação, bem como a definição de um tema padrão para o repositório. A partir do que foi apresentado até aqui podemos considerar que os testes, com possíveis usuários, serão fundamentais para validar todo o trabalho. Estes testes constituem um momento significativo na projetação de um produto, são entendidos como parte do processo de desenvolvimento, objetivando identificar erros, problemas, e consequentemente trazendo melhorias no uso, garantindo uma melhor experiência para o usuário final. Diante dessa observação, destaca-se ainda mais a necessidade de se considerar esse momento, dentro do GODP associado a implantação, como parte crucial para a finalização do repositório.

\section{Textiles Fabris: Visual identity of the digital collection}

\begin{abstract}
The manufacturing complex implemented in Rio Tinto played a significant role in the national textile production chain between the 1920s and 1980s, standing as one of the most important in Brazil. All processes linked to the development of the textile product took place in the factory city itself, which also absorbed European influences in architecture and in the initial implementation of its machinery. Realizing these peculiarities, the theme highlighted here has been the object of study for scientific initiation projects. Over the past six years, the projects have registered the memory of the material heritage originating in the city, which has been undergoing constant processes of de-characterization. In order to disseminate information on the subject and spread the history of this rich Brazilian industrial environment, the online digital repository Têxteis Fabris is being developed to house the results of scientific initiation projects. Through the Tainacan platform, the collection will make available, freely for access and consultation, photographic records of the main elements associated with this industrial moment in the city. That said, the text presented here proposes to address the process of building the visual identity that will be linked to this repository listing the elements and steps necessary for its construction. For this, a design methodology was defined that could manage the development of the identity, as well as the necessary tests for its validation. The results are presented with the logo that should be associated with the repository, icons of the three main themes that surround the collection, patterns that can be associated with images for dissemination, as well as the definition of a standard theme to be followed within Tainacan. As a conclusion, the need to consider the tests to validate all the work performed so far is highlighted.
\end{abstract}

Keywords: Rio Tinto; Têxteis Fabris; Visual identity; 


\section{Referências bibliográficas}

GUNN, P.; CORREIA, T. B. Núcleos autárquicos e fechados. In: PANET, A. et al. Rio Tinto: estrutura urbana, trabalho e cotidiano. João Pessoa: Unipê Editora, 2002.

LAMPERT, Sérgio Renato. FLORES, Daniel. O repositório digital como instrumento para preservação e acesso ao patrimônio arquivístico documental. 70 Simpósio Internacional Memória e

Patrimônio. Pelotas. Rio Grande do Sul. 06 de novembro - 08 de novembro de 2013.

LEHMKUHL, Luciene; SILVA, Jeferson Luiz Brás da. A cidade-fábrica de Rio Tinto na pesquisa em design. In: Anais do 120 Congresso Brasileiro de Pesquisa e Desenvolvimento em Design, 2016, Belo Horizonte. Blucher Design Proceedings. São Paulo: Editora Blucher, 2016. p. 437.

MARTINS, Dalton Lopes; SILVA, Marcel Ferrante; CARMO, Danielle do. Repositório digital com o software livre tainacan: revisão da ferramenta e exemplo de implantação na área cultural com a revista Filme Cultura. 180 Encontro Nacional de Pesquisa em Ciência da Informação. Marília. São Paulo. 2017. Disponível em:

http://enancib.marilia.unesp.br/index.php/xviiienancib/ENANCIB/paper/viewFile/ 472/838 . Acesso em: 22 de Maio de 2020.

MERINO, Giselle Schmidt Alves Díaz. GODP - Guia de Orientação para Desenvolvimento de Projetos: Uma metodologia de Design Centrado no Usuário. Florianópolis: Ngd/ Ufsc, 2016. Disponível em: 〈www.ngd.ufsc.br〉. Acesso em: 15 jul. 2019.

MONTSERRAT. About Montserrat.2017 Disponível em: https://fonts.google.com/specimen/Montserrat\#standard-style\%20s. Acesso em: 31.05.2021

PANET, Amélia et al. Rio Tinto: estrutura urbana, trabalh e cotidiano. 1. ed. UNIPÊ Editora, João Pessoa-PB, 2002.

PASSOS, Jaire Ederson; PASSOS, Paula Caroline Schifino Jardim; DE SOUZA VANZ, Samile Andrea. Projeto de identidade visual para a revista Em Questão. Em Questão, v. 20, n. 2, p. 8-27, 2014.

SCHERER, Fabiano de Vargas; SCHERER, Gabriel Bischoff. Estudo de método projetual para Sistema Integrado de Identidade Visual, design da informação e orientação. InfoDesign: Revista Brasileira de Design da Informação. [São Paulo], SBDI. Vol. 12, n. 2 (2015), p. 171-193, 2015.

RASLAN, Eliane Meire Soares. Posicionamento, identidade e visibilidade da marca. Rizoma, v. 2, n. 1, p. 136-151, 2014.

SILVA, Jeferson Luiz Braz da; LEHMKUHL, Luciene. O Design na Comunicação do Patrimônio. In: Anais do $13^{\circ}$ Congresso Pesquisa e Desenvolvimento em Design (2018). São Paulo: Blucher, 2019. p. 2906- 2917. ISSN 2318-6968, DOI 10.5151/ped2018-4.1_ACO_17

VÁSQUEZ, Ruth Peralta. Identidade de marca, gestão e comunicação. Organicom, v. 4, n. 7, p. 198211, 2007.

VIANA, Cassandra Lúcia de Maya; MÁDERO, Arellano Miguel Ángel; SHINTAKU, Milton. Repositórios institucionais em ciência e tecnologia: uma experiência de customizacão do DSpace., 2005 . In: 30

Simpósio Internacional de Bibliotecas Digitais. São Paulo, 28 november - 02 december 2005.

WORDPRESS. Usando temas. 17 de setembro de 2019. Disponível em:

https://codex.wordpress.org/pt-br:Usando_Temas. Acesso em: 13 de julho de 2020. 